\title{
Design of a CCN Instrument for Airborne Measurement
}

\author{
PATRICK Y. ChUANG \\ Environmental Engineering Science, California Institute of Technology, Pasadena, California \\ Athanasios Nenes \\ Department of Chemical Engineering, California Institute of Technology, Pasadena, California \\ JAMES N. SMith \\ Environmental Engineering Science, California Institute of Technology, Pasadena, California \\ Richard C. Flagan and John H. Seinfeld \\ Department of Chemical Engineering, California Institute of Technology, Pasadena, California
}

(Manuscript received 1 April 1999, in final form 3 October 1999)

\begin{abstract}
A new instrument for measuring cloud condensation nuclei (CCN) on board small aircraft is described. Small aircraft are attractive mainly because they are less costly, but they require instruments that are designed for minimum weight, volume, and power consumption; that are robust; and that are capable of autonomous operation and making measurements at a frequency appropriate for aircraft speeds. The instrument design combines the streamwise gradient technique previously reported by J. G. Hudson, and the alternating gradient condensation nuclei counter described by W. A. Hoppel et al. Field and laboratory measurements, and modeling studies show that this combination exhibits poor sensitivity for the measurement of $\mathrm{CCN}$ spectra; for the climatically important range of critical supersaturations, $0.03 \%-1 \%$, the measured variable, droplet diameter, varies only by $30 \%$. The ability to resolve CCN spectra using this method is therefore in question. Studies of this instrument in a fixed supersaturation mode show that it can measure $\mathrm{CCN}$ at a single supersaturation in the range of $0.1 \%-2 \%$. Calibration and testing of the instrument in this mode is described. The instrument is capable of making accurate, high-frequency $(>0.1 \mathrm{~Hz})$ measurements of $\mathrm{CCN}$ at a fixed supersaturation, while satisfying the constraints for small aircraft.
\end{abstract}

\section{Introduction}

Cloud condensation nuclei (CCN), those particles that nucleate cloud droplet formation, influence the droplet number and size distribution in a cloud, which in turn determine the cloud albedo, lifetime, and precipitation rate, with important climate effects (IPCC 1996; Twomey 1977). Knowledge of the nature of CCN is essential to understanding possible anthropogenically induced climate change.

Ideally, a measurement of cloud condensation nuclei would reveal the distribution of particles with respect to the supersaturation at which they activate in the atmosphere. Unfortunately, no laboratory $\mathrm{CCN}$ measurement to date can faithfully reproduce the atmospheric

Corresponding author address: John H. Seinfeld, Mail Code 21041 Caltech, Pasadena, CA 91125.

E-mail: seinfeld@its.caltech.edu conditions in which cloud droplets form because of the compromises inherent in instrument design. Efforts to measure $\mathrm{CCN}$ in the cloud-forming regions of the atmosphere are especially constrained. An airborne instrument must work within weight, power, and size constraints imposed by the aircraft on which the instrument is to be flown. Moreover, high flight speeds mean that measurements probe substantial distances along the flight path unless the measurement can be completed in a short time.

$\mathrm{CCN}$ are most frequently measured by exposing the aerosol to supersaturation with water vapor for sufficient time that the droplets activate and begin to grow. The supersaturation is achieved by maintaining wetted surfaces that enclose the aerosol sample at different temperatures. Maintaining the surface wetting is difficult, often requiring operator intervention or limiting the operating time of the instrument. In practice, not all CCN are activated as the largest particles (those with the lowest critical supersaturation) are not exposed to super- 
saturated conditions for a duration sufficient for them to grow to their activated diameter (Chuang et al. 1997).

Most measurements are performed by exposing the aerosol to a fixed supersaturation so that the number concentration that activate at supersaturations equal to or lower than the posed value can be determined. The CCN spectrum can be determined by a sequence of measurements at different applied supersaturations. The time required for the completion of one such sequence is long enough that few steps are employed in most airborne measurements.

Hudson (1989) introduced an indirect approach for rapid measurement of supersaturation distributions. In Hudson's method, the aerosol is exposed to a supersaturation that increases with time as the aerosol flows through the supersaturation apparatus. Particles that activate early, when the supersaturation is low, grow for longer time than do those that must wait until the supersaturation has reached higher values. The supersaturation at which the droplet activated is inferred from the ultimate size achieved at the end of the flow channel. Although this measurement method is less direct than the fixed supersaturation technique, and must be calibrated to account properly for the influence of growth kinetics on the size/critical supersaturation relationship, the possibility of measuring all critical supersaturations simultaneously and in short times makes it attractive for airborne measurements. Hudson (e.g., Hudson and Clarke 1992; Hudson and Svensson 1995; Hudson et al. 1998) has used this instrument to measure CCN distributions in a number of measurement campaigns. This instrument is, however, limited to use aboard aircraft that can accommodate its size, weight, and need for operator attention.

We seek to measure CCN using smaller, less costly aircraft. Such a strategy imposes serious constraints on the size, weight, and power consumption and requires robust, unattended operation. Chuang et al. (2000) implemented Hudson's (1989) streamwise gradient approach to $\mathrm{CCN}$ measurement in combination with a novel tubular $\mathrm{CCN}$ design developed by Hoppel et al. (1979) to produce a robust instrument for CCN measurement that was small enough to fly aboard the Center for Interdisciplinary Remotely Piloted Aircraft Studies (CIRPAS) Pelican during the 2nd Aerosol Characterization Exeriment (ACE-2) field campaign. This instrument was used during 15 Pelican flights flown out of Tenerife in the Canary Islands in June and July 1997. Although the instrument was designed to provide supersaturation spectra, the data obtained in the field measurements were deemed to exhibit sufficient detail only to report the number concentration of $\mathrm{CCN}$ at but one supersaturation, $0.1 \%$.

This paper experimentally probes the reasons for the low resolution of this streamwise gradient $\mathrm{CCN}$ spectrometer. The suitability of using this instrument for measurements at fixed supersaturation, as it was used during ACE-2, is also examined. The latter approach is shown to be preferable given the limitations of this instrument. We begin with a brief review of $\mathrm{CCN}$ measurement methods.

\section{Background}

The equilibrium relationship between the diameter of a droplet and the relative humidity of the surrounding air is given by the Köhler equation (Seinfeld and Pandis 1998):

$$
\ln \left(\frac{p_{w}}{p^{o}}\right)=\frac{4 \sigma M_{w}}{R T \rho_{w} D_{p}}-\frac{6 n_{s} M_{w}}{\pi \rho_{w} D_{p}^{3}},
$$

where $p_{w}$ is the vapor pressure of water over a droplet, $p^{o}$ is the vapor pressure of a flat surface of water, $\sigma$ is the droplet surface tension, $M_{w}$ is the molecular weight of water, $R$ is the universal gas constant, $T$ is the droplet temperature, $\rho_{w}$ is the density of liquid water, $n_{s}$ is the number moles of solution-phase species, and $D_{p}$ is the droplet diameter. The Köhler curve exhibits a maximum at a droplet diameter known as the critical diameter, $D_{\mathrm{pc}}$, and at a supersaturation, $S=\left(p_{w} / p^{o}\right)-1$, known as the critical supersaturation, $S_{c}$. When a droplet grows (by condensation of water vapor in the case of clouds) to a diameter larger than the critical diameter, it is considered to be activated, and is able to grow spontaneously to very large sizes, limited only by the kinetics of condensational growth. The activation and subsequent growth of CCN are the first steps in the formation of cloud droplets.

A review of existing $\mathrm{CCN}$ instruments, all of which have been implemented on aircraft platforms, is first presented. The review focuses on the features of each instrument, especially in regard to their utility for aircraft operation. Nearly all airborne CCN data that are in the literature have been acquired using the instruments to be reviewed, although there exist others for which airborne measurements have been reported (e.g., Leaitch and Megaw 1982). The purpose of this review is to summarize the features of existing $\mathrm{CCN}$ instruments, especially with regard to their ability to make airborne measurements.

\section{a. Static thermal diffusion cloud chamber}

$\mathrm{CCN}$ counters that measure $\mathrm{CCN}$ concentration at one supersaturation at a time have existed for decades. The classic static thermal diffusion chamber design consists of two parallel horizontal metal plates whose facing surfaces are wetted (Twomey 1963). When held at different constant temperatures, a nearly parabolic supersaturation profile is achieved between the plates. Those particles that are able to activate at the peak supersaturation in the chamber grow to become large droplets, while those that cannot activate achieve only their equilibrium diameter. The peak supersaturation can be inferred by measuring the temperatures of the two plates 
(or more specifically, the temperature of the surface of the liquid water film that is exposed to the chamber interior). In practice, the peak supersaturation is not perfectly known for a variety of reasons, including the uncertainty in the temperature of the water film.

To measure the CCN spectrum in this type of instrument, the temperature difference between the plates must be systematically changed. This typically requires several minutes. The thermal diffusion chamber is, therefore, limited to low temporal resolution, typically fewer than one measurement per minute (Lala and Jiusto 1977). The lowest supersaturation at which CCN can be measured is generally greater than $0.2 \%$, not low enough to match supersaturations found in some marine stratus clouds. The highest supersaturation is typically at least $2 \%$. Predictions of the uncertainty in CCN concentration measurements in the static thermal diffusion cloud chamber have been estimated to be a factor of two at $1 \%$ supersaturation, increasing to a factor of 10 at $0.1 \%$ supersaturation (Alofs and Carstens 1976). Jiusto et al. (1981) report the agreement among five of the best nine instruments during an intercomparison experiment. The average variability (as measured by $\sigma / \bar{N}$ ) among the devices is 0.24 and 0.42 for $1.0 \%$ and $0.2 \%$, respectively.

Even though these instruments have long been in operation, there are a number of issues in the interpretation of static diffusion $\mathrm{CCN}$ counter data that remain unresolved. One common technique for determining the number concentration of activated droplets in static diffusion cloud chambers is through calibrated photometric measurements of the total intensity of light scattered from the ensemble of droplets within an illuminated view volume. Another popular method involves taking photographs or a video recording of an illuminated view volume and counting the number of imaged droplets. Recent research has shown that the photometric method is unreliable in some circumstances (G. C. Roberts 1999, personal communication). Neither the peak light intensity nor integrated signal area can be unambiguously related to droplet number concentration because both measures are also dependent on the droplet size distribution, which is neither known a priori nor measured by the instrument. Counting droplets using photographs or videotapes of the view volume, while labor- and/or computationally intensive, appears to be more accurate at present.

Another potential data interpretation issue involves the measurement of the temperature of the two plates. The relevant temperatures that determine the supersaturation profile for static diffusion chambers are those of the water films that wet the plates. Typically, however, these instruments measure and control the temperature at a location some distance from the plate surfaces, which could cause the stated supersaturation at which CCN are counted to be overestimated by a significant amount, especially at small temperature differences, that is, low supersaturations.
Detection of sedimenting droplets that were not originally activated in the view volume may also bias CCN measurements. The supersaturation above and below the view volume that is approximately centered between the two plates is lower than that in the view volume, but may be sufficient to activate some particles. Moreover, higher supersaturations may occur before vapor and thermal diffusion reach steady state. Activated droplets can grow sufficiently large to sediment into the view volume in the interval during which the concentration measurement is being made, which could lead to a systematic measurement bias. This is a complex issue for which questions remain unresolved.

On the positive side, static thermal diffusion chambers can be lightweight and robust (Delene et al. 1998). However, issues associated with data interpretation, and the large inherent uncertainties in measurements for supersaturations in the range relevant to marine stratiform clouds, that is, less than $0.5 \%$, suggest that other measurement techniques should be explored in order to facilitate accurate $\mathrm{CCN}$ concentration measurements.

\section{b. Continuous-flow parallel-plate diffusion chambers}

A continuous-flow parallel-plate thermal diffusion chamber has a number of potential advantages over static diffusion counters. Because the sample flow is continuous, the time resolution of the instrument is not limited to that of the batch flow cycle as in static thermal diffusion chambers. Furthermore, the instrument operates at steady state so flow transients that complicate data analysis in static chambers are avoided. In the continuous-flow instrument, a particle-free sheath flow can be used to confine the sample to a region close to the supersaturation maximum so that all $\mathrm{CCN}$ are exposed to the same supersaturation, thereby increasing the precision of the measurement. However, such instruments are subject to some of the same limitations as static chambers. Due to the time required for droplet growth at low supersaturation, the lowest supersaturation at which the measurement can be made is about $0.1 \%$. When the two plates are horizontal, gravitational sedimentation limits the time during which droplets experience a uniform supersaturation. Thermophoretic and diffusiophoretic forces limit the growth time between vertical plates, although this time limit is as much as eight times that for horizontal plates (Sinnarwalla and Alofs 1973). Such instruments have, in the past, also been relatively massive, with typical plate sizes of 0.4 $\mathrm{m} \times 0.3 \mathrm{~m} \times 0.013 \mathrm{~m}$ (Hudson and Squires 1976) and $1.0 \mathrm{~m} \times 0.13 \mathrm{~m}$ (Sinnarwalla and Alofs 1973; plate thickness not reported). In order to measure the $\mathrm{CCN}$ spectrum, the temperature of the plates must be changed, which, given the large heat capacity of the plates, is both time and energy intensive.

An extension of this design, first published by Fukuta and Saxena (1979), utilizes a gradient in temperature transverse to the flow direction to expose particles along 


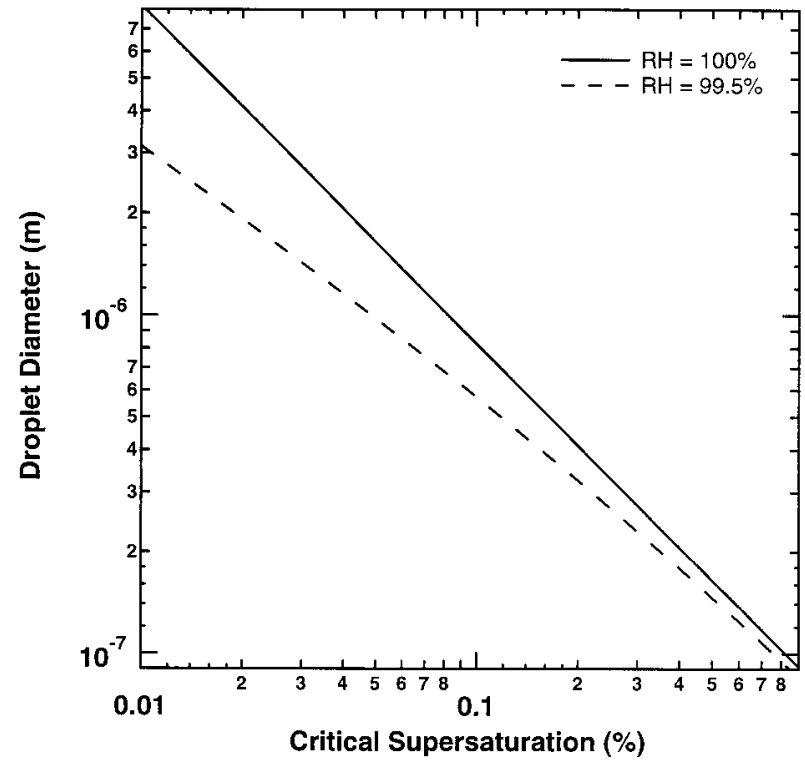

FIG. 1. Equilibrium diameter of droplets at $100 \%$ and $99.5 \%$ relative humidity as a function of particle critical supersaturation.

different streamlines (but still flowing in the plane centered between the two plates) to different uniform supersaturations. Although this principle can be used to measure the $\mathrm{CCN}$ spectrum in near real time, it is still limited in supersaturation range and, in its current form, by size and weight $(0.84 \mathrm{~m} \times 0.23 \mathrm{~m} \times 0.0064 \mathrm{~m}$ and $26 \mathrm{~kg}$ for the chamber alone).

Continuous-flow parallel-plate devices can achieve better time resolution and precision than static thermal diffusion chambers since they are not subject to a batch flow cycle and complex transient supersaturations. Such instruments must be significantly larger than static chambers, however, to minimize edge effects. As a result, they tend to be heavier and consume more power than the smaller diffusion cloud chambers.

\section{c. Isothermal haze chambers}

Isothermal haze chambers (IHCs), first proposed and built by Laktionov (1972), differ from other CCN instruments in that they do not activate droplets, but rather measure the diameter of particles in equilibrium with a saturated atmosphere (relative humidity of 100\%). Köhler theory is then used to deduce the critical supersaturation of the particle from its equilibrium size. In practice, it is difficult to maintain exactly $100 \% \mathrm{RH}$, and the measurement is highly sensitive to deviations from $100 \%$ RH (Fig. 1). If the RH is $99.5 \%$, for example, rather than exactly $100 \%$, it can be seen from Fig. 1 that a systematic overestimation of each particle's critical supersaturation results. This overestimation is about $20 \%$ for particles with $S_{c}$ of $0.2 \%$, increasing to more than a factor of two for particles with $S_{c}$ of $0.04 \%$. Alternately, this systematic bias can also be interpreted as resulting in an underestimate of the cumulative $\mathrm{CCN}$ concentration at a given $S_{c}$. For example, the concentration of droplets larger than $0.5 \mu \mathrm{m}$ in diameter is the concentration of $\mathrm{CCN}$ with $S_{c}$ less than approximately $0.17 \%$; if an IHC actually operates at $99.5 \% \mathrm{RH}$, the measured concentration of $\mathrm{CCN}$ would be those with $S_{c}$ less than $0.12 \%$, resulting in an underestimation of the CCN concentration at $0.17 \%$. One solution for this problem would be to calibrate the instrument with $\mathrm{CCN}$ of known $S_{c}$. If the $\mathrm{RH}$ in the instrument is stable over time, then the correct relationship between droplet diameter and $S_{c}$ could be established at this RH. A more detailed analysis of IHCs is presented by Fitzgerald et al. (1981).

One advantage of such an instrument is that measurements of the size distribution of the outlet droplets reveals the range of measurable critical supersaturations of the input aerosol simultaneously. Another advantage is that it is able to probe critical supersaturations lower than those of most other instruments (though subject to increasing uncertainties as supersaturation decreases as described above). IHCs have been operated as low as $0.016 \% S_{c}$ (Alofs 1978). Current IHCs are, however, limited to supersaturations no higher than $0.25 \%$ because the optical particle counters used for droplet sizing cannot detect particles less than $0.16 \mu \mathrm{m}$ in diameter. That minimum size corresponds to the equilibrium size for a salt particle with $S_{c}=0.25 \%$. If the measurement range could be extended to smaller particle sizes, the upper limit in critical supersaturation could be increased. Although the instrument itself is fairly compact [e.g., the instrument of Alofs (1978) was $5.5 \mathrm{~cm}$ in diameter and $30 \mathrm{~cm}$ long], the low sample rate required to achieve equilibrium without causing significant water vapor depletion limits the count rate and may cause poor counting statistics in clean environments.

Perhaps the most important limitation, however, is that IHCs do not, strictly speaking, measure CCN since droplets are not activated. In theory, this is not a problem if the particles are composed of completely soluble, surface-inactive compounds since the particle $S_{c}$ can be uniquely derived from its equilibrium size at $100 \% \mathrm{RH}$. However, the results become ambiguous if slightly soluble compounds or surfactants are present in the particles. Such substances can change droplet diameter at $100 \%$ RH but not cause changes in $S_{c}$ (Alofs 1978; Shulman et al. 1996). Such issues are likely to be more important in cases where substantial amounts of organics are present.

IHCs are attractive primarily because of their small size, their ability to probe a range of critical supersaturations simultaneously, and their ability to measure CCN with very low critical supersaturations. The main drawbacks are potentially large uncertainties in the relationship between droplet diameter and critical supersaturation, and the fact that they do not measure activated $\mathrm{CCN}$. Whether or not these drawbacks do cause measurement problems remains an open question. 


\section{d. Streamwise gradient CCN spectrometer}

Hudson (1989) modified a continuous-flow thermal diffusion chamber so that the supersaturation that particles experience increases with time as they flow through the instrument. Particles that activate at low supersaturations near the entrance to the instrument have more time to grow, and experience a larger driving force due to a lower equilibrium vapor pressure, than do those that activate at higher supersaturations (closer to the outlet of the instrument). The CCN spectrum is inferred from measurements of the size distribution of the droplets at the instrument outlet. Since this method measures the end result of particle growth in a time-varying environment, it must be calibrated to relate outlet droplet diameter to inlet $S_{c}$. The range of measurable critical supersaturations is reported to be much larger than that for other thermal diffusion chambers, about $0.01 \%-1 \%$, covering much of the range of interest for climatically important warm clouds. The size of Hudson's chamber is comparable to previous continuous-flow thermal diffusion instruments. As in the isothermal haze chamber measurements, in principle the $S_{c}$ distribution can be obtained simultaneously for all $S_{c}$, a desirable feature for aircraft studies. Moreover, in contrast to IHCs, most of the measured droplets are activated. For these reasons, the instrument developed for use aboard the $\mathrm{Pel}$ ican (with a total instrument payload of $150 \mathrm{~kg}$ ) was based on the streamwise gradient concept. However, the size and weight of Hudson's instrument are prohibitive for small aircraft such as the Pelican, and therefore a totally different physical implementation was selected.

\section{e. Spectrometer data analysis}

It is necessary to invert raw data from any spectrometer to produce a spectrum. In practice, this is likely to be nontrivial for streamwise-gradient CCN spectrometers (and perhaps IHCs as well). Even particles with identical critical supersaturations produce a distribution of optical signals, so an inversion routine that accounts for this distribution of responses is needed to infer the CCN spectrum from the optically measured size distribution. This inversion problem is analogous to that encountered in the interpretation of differential mobility analyzer data, and can be described by the integral equation

$$
\int_{a}^{b} K_{i}\left(S_{c}\right) N\left(S_{c}\right) d S_{c}+\varepsilon_{i}=y_{i}, \quad i=1 \text { to } m,
$$

which can be approximated by

$$
\sum_{j=1}^{n} K_{i}\left(S_{c, j}\right) N\left(S_{c, j}\right)+\varepsilon_{i}=y_{i}, \quad i=1 \text { to } m
$$

where $K_{i}$ is the instrument kernel function, $N$ is the critical supersaturation distribution (the desired quantity), $y_{i}$ is the measured variable (in this case, measured light scattering intensity), $\varepsilon_{i}$ is the error associated with each measurement $y_{i}, m$ is the number of measurement channels (in this case, the number of different light intensities measured), and $n$ is the number of discrete values into which the $S_{c}$ interval $[a, b]$ is divided. For this instrument, the kernel function describes the measured distribution of outlet signals for a sample containing particles of only one $S_{c}$ and this function is determined experimentally through calibration. Solving Eq. (2) is an ill-posed problem for which an infinite number of solutions is possible (e.g., Kandlikar and Ramachandran 1999). Smoothing requirements are typically imposed to constrain the solution.

If, instead of solving Eq. (2), each output signal is assumed to correspond uniquely and exactly to one inlet $S_{c}$, the inferred CCN spectrum is broadened and flattened because the instrument transfer function is not an ideal delta function. To better understand this, imagine an experiment where a perfectly monodisperse, or delta function, distribution was measured by a nonideal $\mathrm{CCN}$ spectrometer, that is, a spectrometer whose transfer function is not a delta function but rather a distribution with some finite width. The resulting measured droplet size distribution would be exactly this transfer function. If, when inverting this measured spectrum, the instrument is assumed to be ideal, then the inferred CCN spectrum would be that transfer function, which is flatter and broader than the original input distribution, which was a delta function. The accuracy of the inversion of droplet size distributions resulting from the streamwisegradient technique to produce $\mathrm{CCN}$ spectra has not been adequately addressed in the literature, and, as we show below, will prove to be a significant limitation of the instrument described here.

\section{Instrument design}

The instrument described here uses the streamwise gradient technique described by Hudson (1989), but differs in the method by which the supersaturation profile is imposed. The goal was to build a robust and automated instrument with minimum mass, volume, and power consumption that is suitable for unattended operation on small aircraft, and that makes high quality measurements at high frequency simultaneously for a wide range of supersaturations. The final instrument has an electronics box with a volume of about $33 \mathrm{~L}$ plus a column $0.7 \mathrm{~m}$ tall $\times 0.1 \mathrm{~m}$ diameter (additional $5 \mathrm{~L}$ ), weighs $14 \mathrm{~kg}$, and consumes $180 \mathrm{~W}$ of power.

\section{a. CCN column}

The CCN column is the part of this spectrometer that establishes the supersaturation profile that allows the aerosol to activate and grow to large $(>1 \mu \mathrm{m})$ size. The column is based on a condensation nuclei counter design of Hoppel et al. (1979) that employed a wet-wall tubular column that is divided into alternating hot and cold 


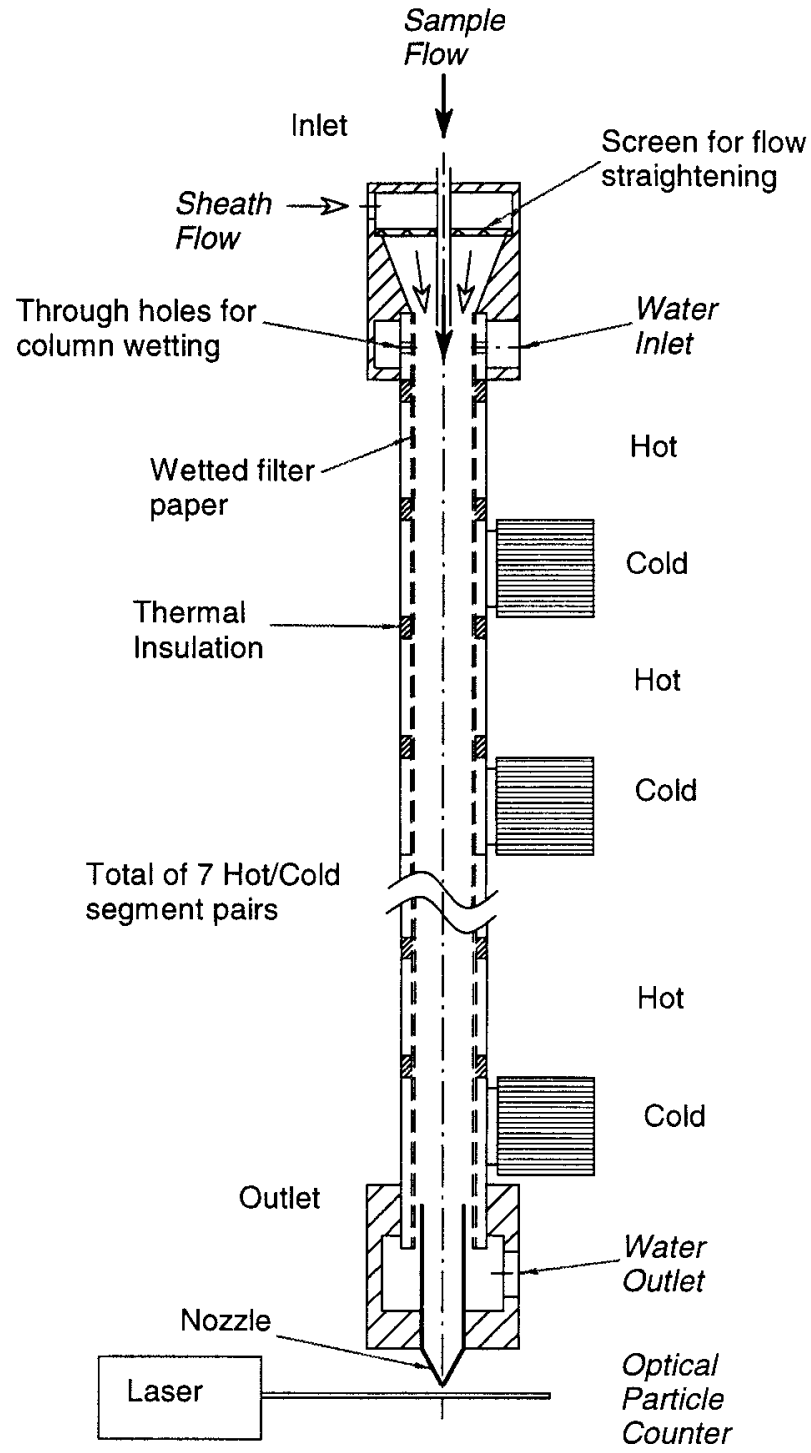

FIG. 2. Schematic of wet-walled column for $\mathrm{CCN}$ instrument. Thermistors are embedded vertically into the walls of each segment at a position that is equidistant from the inner and outer surfaces.

segments to generate a supersaturation profile. By saturating the airstream at the hot temperature, and then exposing it to the cold temperature, a supersaturation is generated. The supersaturation is maximum on centerline. The temperatures of the successive segments and gas flow rate can be adjusted to generate a supersaturation profile that reaches a fixed maximum (fixed temperature difference between all hot and cold segments), or one that on average is continuously increasing (increasing temperature difference between successive hot and cold segments).

The instrument is illustrated in Fig. 2. A thin-walled stainless steel tube $(19.0 \mathrm{~mm}$ OD $\times 0.25 \mathrm{~mm}$ wall thickness) serves as the droplet growth section. Alternating sections of "hot" and "cold" aluminum segments are slip fit over the outside of this tube. Each of the segments is $28 \mathrm{~mm}$ tall, square ( $25 \mathrm{~mm}$ on a side) in cross section, and separated from adjacent sections by a 6.4-mm-thick insulation layer. To maximize thermal conduction between these segments and the tube, thermal compound (Wakefield 120-8) is used. Hot sections are heated using resistance heaters and the cold sections are cooled with thermoelectric coolers. To maximize the efficiency of the coolers, a fan at the bottom of the column blows air through a duct enclosing heat sinks attached to the cold sections. A shimstock duct constrains this airflow. All sides of the column segments, except for the heat sinks, are insulated to minimize losses. Each segment has a nominal $20 \mathrm{k} \Omega$ (measured at $25^{\circ} \mathrm{C}$ ) thermistor embedded in it to measure the segment temperature. The interior of the stainless tube is lined with wetted filter paper (Whatman 3Chr).

The aerosol sample is introduced into the column along its centerline and is surrounded by a particle-free sheath flow that constrains the sample flow. Typical operating flow rates are a total flow rate (sheath + sample) of $1.67 \times 10^{-5} \mathrm{~m}^{3} \mathrm{~s}^{-1}(1 \mathrm{lpm})$, and a sheath to sample flow rate ratio of 5 to 1 , yielding an average residence time of $9 \mathrm{~s}$. Particles flowing along streamlines that differ in their radial distance from the column centerline experience different supersaturation profiles. Model simulations (described in section 4a) show that for this flow rate ratio, the ambiguities that result from this effect are small compared to the resolution of the droplet diameter measurement.

The inlet assembly also provides a path for water to flow to the top of the column to wet the filter paper. The column outlet is a 9.5 -mm-diameter thin walled tube that tapers down to a nozzle $1 \mathrm{~mm}$ in diameter. The aerodynamically focused droplets are then optically detected as described below. In this way, measurement of the droplet diameter occurs as close to the column exit as possible, minimizing the possibility of any changes in droplet size.

\section{b. Optical particle counter}

To minimize instrument weight and to measure particle diameter accurately, a new optical particle counter (OPC) was designed specifically for measuring water droplets in the size range 1-20 $\mu \mathrm{m}$ (Fig. 3). The output from a ruggedized laser diode module coupled to a fiberoptic cable (Blue Sky FBC011-00) is expanded to produce a beam that is approximately $4 \mathrm{~mm}$ wide and 0.5 $\mathrm{mm}$ high. This output beam cross section was chosen to minimize the probability of two droplets passing through the beam simultaneously, while maximizing the uniformity of the intensity seen by particles passing through slightly different parts of the beam. The sample flow is accelerated through a nozzle (half angle $15^{\circ}$, output diameter $1 \mathrm{~mm}$ ) and passes through the beam 1 $\mathrm{mm}$ after leaving the nozzle. Ideally, the nozzle tip is as small as possible so that the droplets all pass through 


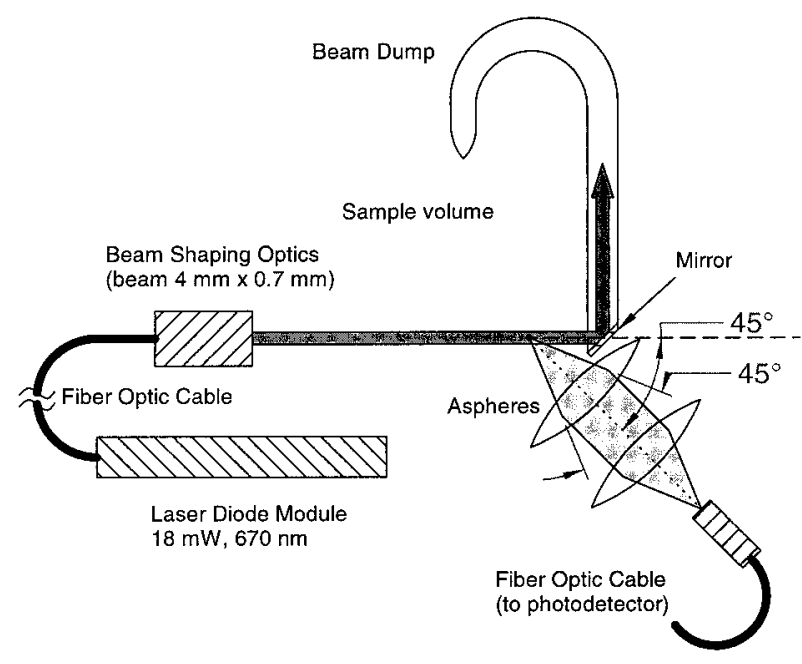

FIG. 3. Optical particle counter schematic.

the same part of the laser, but it was found that tip diameters significantly smaller than $1 \mathrm{~mm}$ resulted in droplet impaction. The scattered light is collected at an axis $45^{\circ}$ off forward and over a solid angle of $0.48 \mathrm{sr}$ (a cone subtended by $45^{\circ}$ total angle) using an aspheric lens. This light is then refocused using an identical aspheric lens onto a fiber optic (1-mm core) that transmits the scattered light to a photomultiplier tube that converts the light intensity to a voltage signal. An avalanche photodiode detector (Hamamatsu C5460-01) has also been used for the same purpose with success. A multichannel analyzer (EG\&G Ortec Trump-2k) card measures the peak height of each voltage pulse generated as droplets pass through the laser beam, and bins that peak height into one of 2048 linearly spaced bins between 0 and $10 \mathrm{~V}$, that is, in roughly 5 -mV-wide bins. As can be seen from Fig. 3, the collection optics intersect the main beam. To solve this problem, a mirror was mounted onto the bottom of a tube, allowing the unwanted main beam to reflect down the axis of the circularly bent black tube and thereby be attenuated.

Using a Mie-scattering code [FORTRAN program bhmie.for from Bohren and Huffman (1983)], an OPC with this configuration was simulated. The theoretically predicted response of the OPC for water $(n=1.33)$ and diethyl sebacate (DES, $n=1.436$ ) droplets is shown in Fig. 4. Also shown are experimental calibration data obtained using DES droplets generated from a Berglund-Liu vibrating orifice aerosol generator (Berglund and Liu 1973). The agreement between theory and experiment is good. The OPC design was chosen such that its response for water droplets is monotonic and does not exhibit any ambiguities due to Mie resonances, which are, for example, present for the DES curve between 1.5 and $2 \mu \mathrm{m}$. These calibration data also show that the resolution of the OPC is about $10 \%$ of the mean diameter (full width at half maximum).

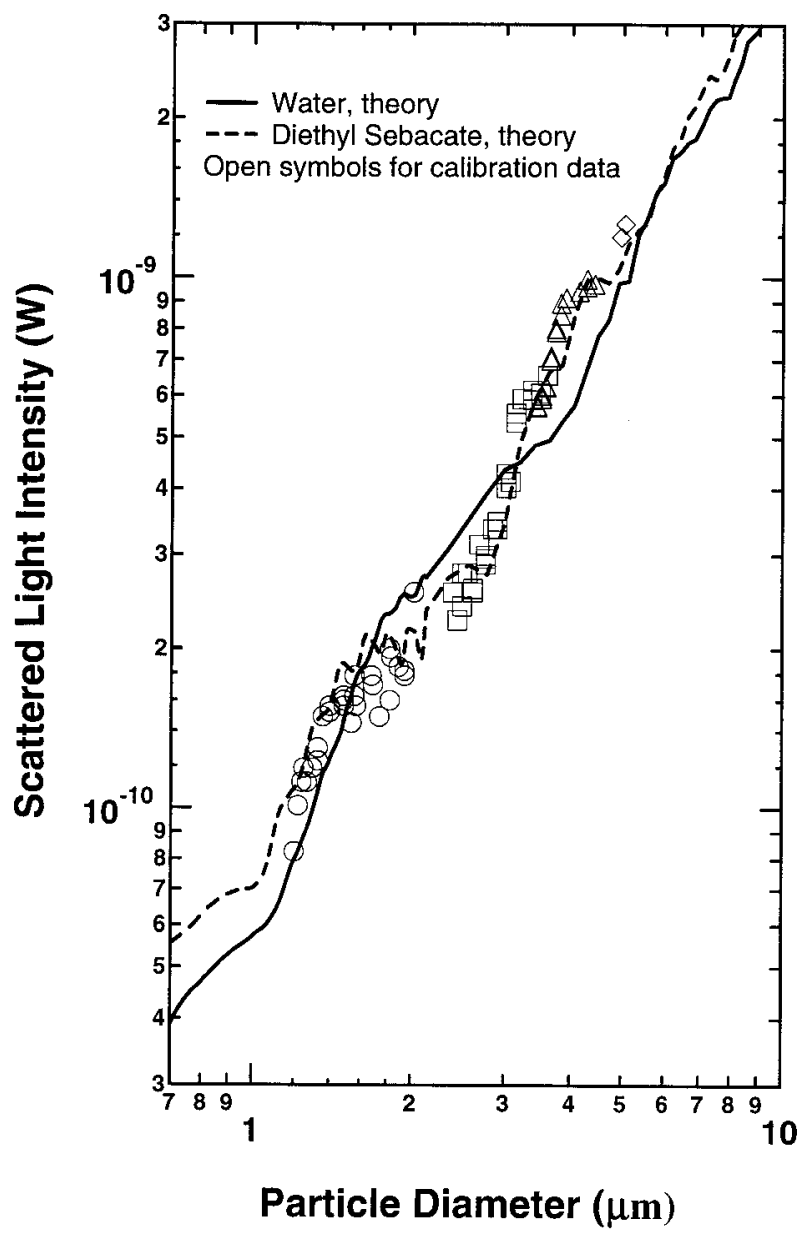

FIG. 4. Comparison of theoretical and measured OPC response. The open symbols are experimental data for diethyl sebacate (DES) droplets.

\section{c. Flow scheme}

A flow diagram of the instrument is presented in Fig. 5. The total flow is controlled with a critical orifice, and the sheath flow rate is controlled with a mass flow controller (Sierra Instruments 840). The aerosol flow is then the difference between the total and sheath flows, and is monitored by measuring the pressure drop across a laminar flow tube. The instrument is operated at ambient pressure. The diffusivities of heat and water vapor in air are pressure dependent; this dependence must be considered in the data analysis. Also, since the mass flow rate of the sheath gas is controlled, the sheath volumetric flow rate increases with decreasing pressure. These variations are accounted for in the data analysis.

The column is wetted before each flight. Because almost all of the water loss in the column is due to evaporation to the gas phase and not to condensed water in the aerosol phase, the sheath flow is humidified to an RH of about $97 \%$. We have found that column performance remains constant for periods over $6 \mathrm{~h}$ without further wetting. A peristaltic pump connected to a water 


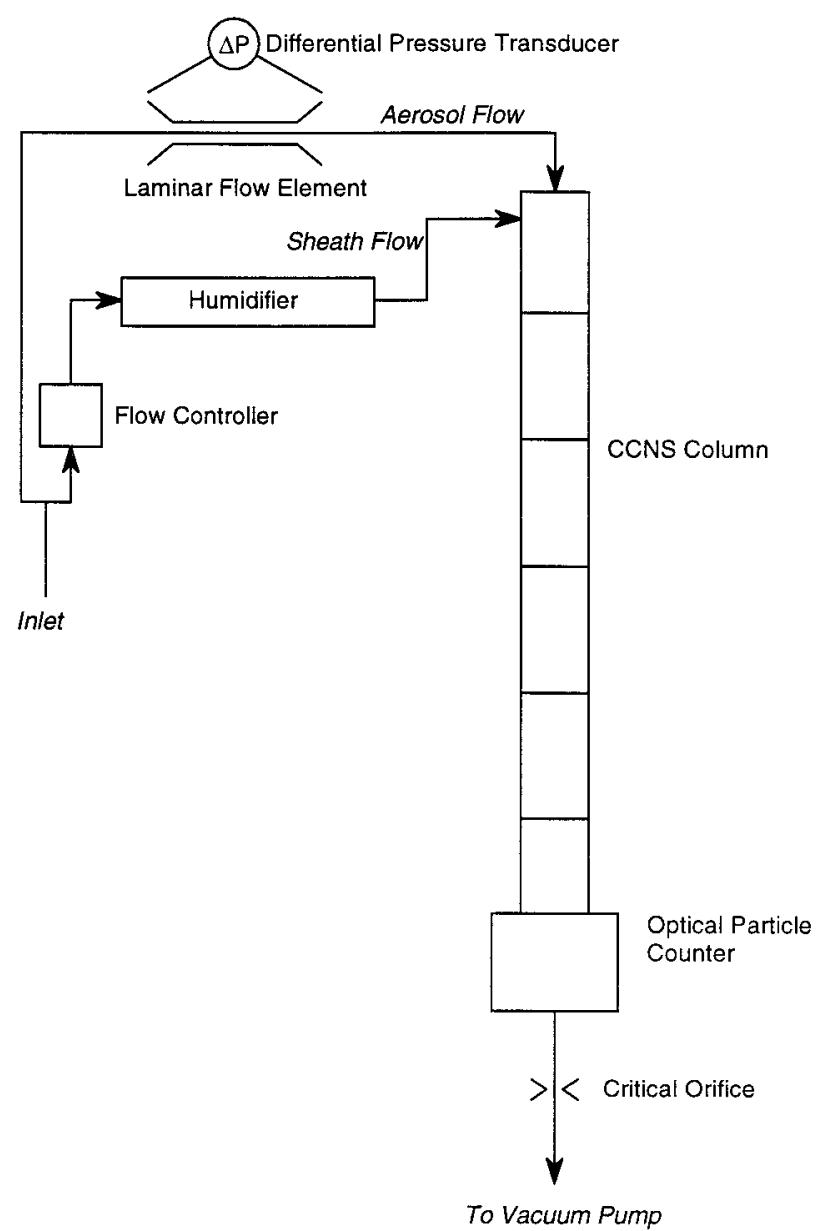

FIG. 5. CCN instrument flow schematic.

reservoir is used to periodically wet the column as necessary.

\section{d. Instrument electronics}

Temperature control of each segment of the column is achieved using a small, lightweight analog proportional-integral controller (Wavelength Electronics RHM-4000). Acquisition of the temperature, flow rate, and pressure data is done using a data acquisition card (National Instruments AT-MIO-64E). The multichannel analyzer (MCA) card and the data acquisition board are both run using a PC/104 half-sized computer (Advantech 386SX-33) with $16 \mathrm{MB}$ of flash memory using the MS-DOS operating system. The data acquisition and control software was developed using the National Instruments LabWindows application. Instrument status data (column segment temperatures and aerosol and sheath flow rates) are acquired and sent to the aircraft onboard computer once per second. Approximately once per minute, the status data stream is interrupted, and the data acquired by the MCA over the past $60 \mathrm{~s}$ are read by this program and sent to the aircraft computer, and the MCA board is reinitialized and acquisition of a new droplet spectrum starts.

\section{Predicted and observed instrument performance}

A model has been developed to simulate the performance of a variety of $\mathrm{CCN}$ instruments, including that described in the present work, in order to aid in understanding the effects of changes in instrument operating parameters, such as the temperature profile.

\section{a. CCN instrument model}

The gas phase is treated as a continuum, so differential momentum, mass, and heat balances are written for a fixed Eulerian grid. A Lagrangian approach is used for describing the evolution of aerosol particles; each particle is tracked as it grows inside the $\mathrm{CCN}$ instrument. The model assumes the following.

1) The flow is at steady state and $2 \mathrm{D}$ axisymmetric

2) Each particle follows a single streamline at the streamline velocity

3) Sedimentation, coagulation, and Brownian diffusion are neglected

4) Walls act as a perfect sink/source of water vapor

\section{1) Governing equations}

With these assumptions, the following system of equations is solved. The continuity equation for the gas phase:

$$
\frac{\partial}{\partial z}(r \rho u)+\frac{\partial}{\partial r}(r \rho v)=0 .
$$

The momentum equation in the radial direction for the gas phase:

$$
\begin{gathered}
\frac{\partial}{\partial z}(r \rho u v)+\frac{\partial}{\partial r}(r \rho v v)-\frac{\partial}{\partial z}\left(r \mu \frac{\partial v}{\partial z}\right)-\frac{\partial}{\partial r}\left(r \mu \frac{\partial v}{\partial r}\right) \\
=-r \frac{\partial p}{\partial r}+r \frac{\partial}{\partial z}\left(\mu \frac{\partial u}{\partial r}\right)+\frac{\partial}{\partial r}\left(r \mu \frac{\partial v}{\partial r}\right)-\frac{\mu v}{r}
\end{gathered}
$$

The momentum equation in the axial direction for the gas phase:

$$
\begin{gathered}
\frac{\partial}{\partial z}(r \rho u u)+\frac{\partial}{\partial r}(r \rho v u)-\frac{\partial}{\partial z}\left(r \mu \frac{\partial u}{\partial z}\right)-\frac{\partial}{\partial r}\left(r \mu \frac{\partial u}{\partial r}\right) \\
=-r \frac{\partial p}{\partial z}+r \frac{\partial}{\partial z}\left(\mu \frac{\partial u}{\partial z}\right)+\frac{\partial}{\partial r}\left(r \mu \frac{\partial v}{\partial z}\right)+\Phi_{\text {buoyancy }}
\end{gathered}
$$

where $r$ and $z$ are the radial and axial coordinates, $u$ and $v$ are the axial and radial components of velocity, $p$ is the pressure, $\mu$ is the viscosity, and $\rho$ is the density. The last term on the rhs of Eq. (6) represents a momentum source due to thermal buoyancy, which for ideal gases is 


$$
\Phi_{\text {buoyancy }}=-\rho g\left[\frac{T-T_{\text {bulk }}(z)}{T_{\text {bulk }}(z)}\right],
$$

where $T_{\text {bulk }}(z)$ is the radially averaged temperature at axial position $z$. Although buoyancy is not expected to be a significant momentum source, it is included for completeness.

The gas phase energy equation is

$$
\begin{aligned}
& \frac{\partial}{\partial z}(\rho u T)+\frac{\partial}{\partial r}(\rho v T)-\frac{k_{a}}{c_{p}} \frac{\partial^{2} T}{\partial z^{2}}-\frac{k_{a}}{c_{p}} \frac{1}{r} \frac{\partial}{\partial r}\left(r \frac{\partial T}{\partial r}\right) \\
& \quad=\frac{\Delta H_{\text {vap }}}{c_{p}} \Phi_{\text {cond }},
\end{aligned}
$$

where $k_{a}$ is the thermal conductivity of air, $c_{p}$ is the heat capacity for moist air, and $\Delta H_{\text {vap }}$ is the enthalpy of vaporization of water. Here $\Phi_{\text {cond }}$ is the rate of condensation of liquid water on the aerosol particles and it is calculated from the droplet growth equation (10) below. Note that use of this term couples the water vapor field with aerosol condensational growth.

Conservation of water vapor is described by

$$
\frac{\partial}{\partial z}(u C)+\frac{\partial}{\partial r}(v C)-D_{v} \frac{\partial^{2} C}{\partial z^{2}}-D_{v} \frac{1}{r} \frac{\partial}{\partial r}\left(r \frac{\partial C}{\partial r}\right)=\Phi_{\text {cond }},
$$

where $C$ is molar water vapor concentration.

A Lagrangian reference frame is used for simulating the particles as they flow through the CCN instrument. The rate of change of droplet diameter $\left(D_{p}\right)$ for each of the particles is calculated from the diffusional growth equation using (Seinfeld and Pandis 1998)

$$
\frac{d D_{p}}{d t}=\frac{1}{D_{p}} \frac{S_{v}-S_{v}^{\mathrm{eq}}}{\frac{\rho_{w} R T}{4 p^{o}(T) D_{v}^{\prime} M_{w}}+\frac{\Delta H_{\mathrm{vap}} \rho_{w}}{4 k_{a}^{\prime} T}\left(\frac{\Delta H_{\mathrm{vap}} M_{w}}{R T}-1\right)},
$$

where $p^{o}(T)$ is the saturation vapor pressure at temperature $T, S_{v}$ is the local saturation ratio, $S_{v}^{\mathrm{eq}}$ is the equilibrium saturation ratio of the droplet, and $R$ is the universal gas constant. Here $D_{v}^{\prime}$ is the diffusivity of water vapor in air modified for noncontinuum effects

$$
D_{v}^{\prime}=\frac{D_{v}}{1+\frac{2 D_{v}}{a_{c} D_{p}}\left(\frac{2 \pi M_{w}}{R T}\right)^{1 / 2}},
$$

where $a_{c}$ is the mass accommodation coefficient. Here $k_{a}^{\prime}$ is the thermal conductivity of air modified for noncontinuum effects

$$
k_{a}^{\prime}=\frac{k_{a}}{1+\frac{2 k_{a}}{a_{T} D_{p} \rho c_{p}}\left(\frac{2 \pi M_{a}}{R T}\right)^{1 / 2}},
$$

where $M_{a}$ is the mean molar mass of air, $a_{T}$ is the thermal accommodation coefficient. The equilibrium saturation ratio of the droplet $S_{v}^{\text {eq }}$ is given by the Köhler equation, Eq. (1), where $S_{v}^{\mathrm{eq}}=p_{w} / p^{o}$.

From these equations (10)-(12), $\Phi_{\text {cond }}$ is then calculated by

$$
\begin{aligned}
\Phi_{\text {cond }} & =\int_{0}^{\infty} \frac{N\left(D_{p}\right) \rho_{w}}{M_{w}} \frac{d V}{d t} d D_{p} \\
& =\int_{0}^{\infty} \frac{N\left(D_{p}\right) \rho_{w} \pi}{2 M_{w}} D_{p}^{2} \frac{d D_{p}}{d t} d D_{p},
\end{aligned}
$$

where $N\left(D_{p}\right)$ is the aerosol size distribution.

\section{2) BOUNDARY AND INITIAL CONDITIONS}

For velocity distribution, two types of initial conditions are considered, either fully developed (i.e., parabolic) or plug flow. Near the wall, a no-slip boundary condition is assumed, $u=v=0$ and for the outlet, $\partial u / \partial z=0$ and $v=0$. Finally, at the symmetry axis, $\partial u / \partial r=\partial v / \partial r=0$.

For temperature, the inlet conditions are prescribed. At the wall, a constant temperature boundary condition is posed for each temperature-controlled segment, while a no heat-flux condition is posed for the insulation sections separating the temperature-controlled sections. At the outlet, $\partial T / \partial z=0$, while at the symmetry axis, $\partial T / \partial r$ $=0$.

For water vapor concentration, the inlet conditions are prescribed based on the inlet relative humidity. At the wall, the air is assumed to be at saturation at the wall temperature. For the outlet, $\partial C / \partial z=0$, while at the symmetry axis, $\partial C / \partial r=0$.

\section{3) Numerical solution of Mathematical MODEL}

The model equations cannot be solved analytically, so a numerical solution is obtained by the finite volume method using an upwind differencing scheme (Patankar 1980). A staggered grid is employed in the calculations such that each velocity grid node lies between two scalar volumes. This procedure ensures that the numerical solution is consistent with respect to pressure. The SIMPLE iterative solution method is used to solve the hydrodynamical cycle of the finite volume equations, while the particle growth equations are solved using the LSODE solver of Hindmarsh (1983). The computer code used for the numerical simulations was based on a modified version of the TEACH-2E code (Gosman and Ideriah 1976). Finally, the numerical solution was obtained using 100 cells for each of the $z$ and $r$ directions.

\section{b. Streamwise gradient operation}

The measured outlet droplet diameter is shown in Fig. 6 as a function of critical supersaturation for an ammonium sulfate aerosol. The measured droplet diameter 


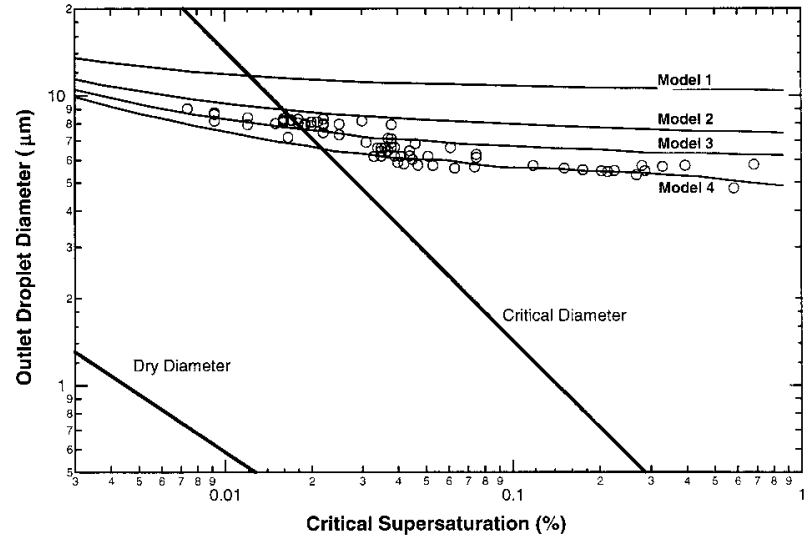

FIG. 6. Model predictions and experimental data relating droplet diameter at the outlet of the CCN column as a function of critical supersaturation for the $\mathrm{CCN}$ instrument in streamwise gradient mode. Models 1-4 use different temperature ramps (Fig. 7). Also shown is the critical diameter (droplets larger than the critical diameter are activated), and the aerosol dry diameter (chemical composition assumed to be pure ammonium sulphate), as a function of critical supersaturation. Open circles are measured calibration data.

exceeds the critical diameter for $S_{c} \gtrsim 0.02 \%$. For lower critical supersaturations, the droplet diameter remains smaller than the critical diameter, indicating that the residence time in the column is insufficient to reach the very large critical diameters. Model predictions are also shown in Fig. 6. Model 1, corresponding to the measured hot and cold segment temperatures, significantly overpredicts the final droplet size. This is not surprising since the model assumes that each wall segment is isothermal at the temperature measured in the aluminum blocks. The temperature difference between the block and the stainless steel tube, and between the tube and the inside water surface, are not taken into account. Unfortunately, they also cannot be measured without perturbing the flows and heat transport. The axial distributions of temperatures along the length of the segments have also been neglected. To compensate for this limitation, calculations were also performed for reduced wall temperature differences as shown in Fig. 7. The experimental data agree well with model 4 for high $S_{c}$, but for lower $S_{c}$, the agreement is better for model 3 . The centerline supersaturation profile corresponding to model 1 is also shown in Fig. 7.

The most notable feature in the growth data shown in Fig. 6 is that, over the large range in $S_{c}$ shown $(0.003 \%-1 \%)$, the outlet droplet diameter changes by at most a factor of two (model 4). Extremely precise measurements of outlet diameter would be required to resolve particles with substantial differences in $S_{c}$, particularly in the climatically important $S_{c}$ range of $0.03 \%-1 \%$ where a factor of 30 difference in $S_{c}$ must be resolved through measurements of a change in diameter of about $30 \%$. The small difference of final droplet size is the result of rapid growth immediately following activation that quickly decelerates as the parti-

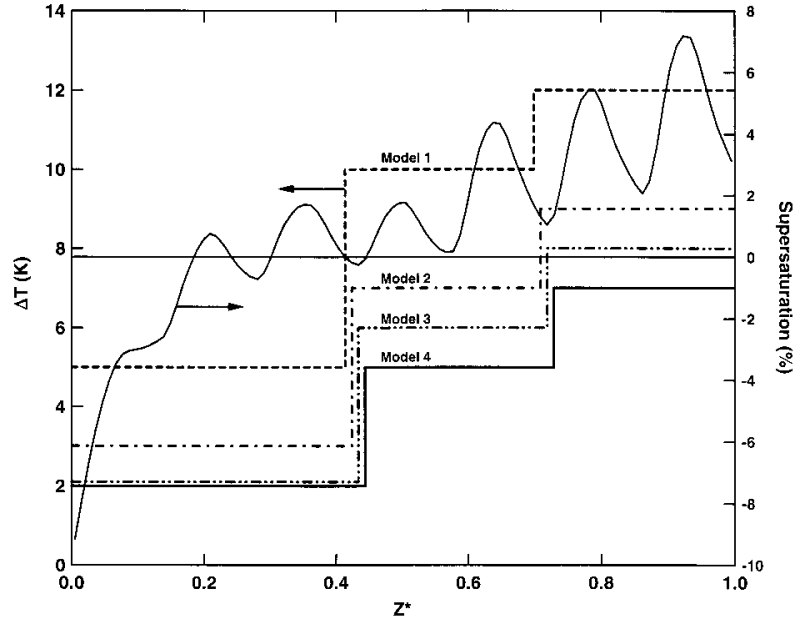

FIG. 7. Temperature difference profiles (left axis) assumed for simulation runs model 1-4, and supersaturation profile (right axis) on centerline for model 1 assuming inlet $\mathrm{RH}$ of $90 \%$, as a function of nondimensionalized distance along the tube $z^{*}$, where $z=0$ and $z$ $=1$ are the inlet and outlet, respectively. All hot temperature segments were held at $295 \mathrm{~K}$, and the cold segment temperatures were changed to impose the temperature differences shown. Some of the temperature profiles are offset slightly from each other for presentation clarity; the true profiles change temperature at identical $z^{*}$.

cles grow, causing newly activated particles to "catch up" with those activated much earlier. Since the rate of condensational growth, $d D_{p} / d t$, is inversely proportional to diameter $D_{p}$, particles that start off smaller grow more quickly than larger ones, which causes droplets to converge to similar sizes.

More quantitatively, using propagation of errors, it can be shown that

$$
\begin{aligned}
& \sigma_{\log S_{c}}^{2}=\left(\frac{d \log S_{c}}{d \log D_{p}}\right)^{2} \sigma_{\log D_{p}}^{2} \text { or } \\
& \sigma_{\log S_{c}}=\left|\left(\frac{d \log S_{c}}{d \log D_{p}}\right) \sigma_{\log D_{p}}\right| .
\end{aligned}
$$

Given that $\sigma_{\log D_{p}}$ is 0.10 (from the resolution estimate in section $3 \mathrm{~b}$ ), and assuming a factor of 30 change in $S_{c}$ for a factor of 1.3 change in $D_{p}, \sigma_{\log S_{c}}$ is calculated to be 1.3. This implies that inferred values of $S_{c}$ are accurate to within a factor of 2.3 due to small random errors in the measurement of $D_{p}$.

Despite the great attention devoted to maximizing the potential of the current instrument, particularly the OPC, the inherent lack of sensitivity in the diameter measurement to $S_{c}$ precludes accurate inversion of the size distribution to produce $S_{c}$ distributions. However, the instrument can still be effectively used as a single supersaturation counter, as discussed below.

\section{c. Airborne CCN measurements}

The streamwise gradient instrument was flown onboard the CIRPAS Pelican during the ACE-2 (Raes et 
al. 2000) during June and July 1997. The results are reported by Chuang et al. (2000). At the time, it was expected that $\mathrm{CCN}$ spectra would be measured using this instrument. Subsequent processing of the data showed that the data could not be inverted reliably to produce spectra, in large part because of the reasons discussed above. It was, however, determined that $\mathrm{CCN}$ concentration at $0.1 \%$ supersaturation could be measured with a high degree of confidence. The instrument exhibited stable operation for 15 flights with an average duration of $5 \mathrm{~h}$, and a maximum duration of $10 \mathrm{~h}$ while taking data at a rate of one measurement per minute. Intercomparison of the $\mathrm{CCN}$ measurements with $\mathrm{CCN}$ instruments on board two other aircraft, Meteo-France Merlin-IV (a static thermal diffusion counter similar to that described by Delene et al. 1998) and the MRF C-130 (also a static thermal diffusion counter), showed good agreement. Overall, the ratio of Pelican to Merlin CCN concentrations was $1.15 \pm 0.15(1 \sigma)$, which represents over $360 \mathrm{~min}$ of Pelican data at ata rate of one measurement per minute. The MRF C-130 and Pelican were in close proximity for a 15 -min period on 14 July, during which the present and MRF instruments measured CCN concentrations of $57 \mathrm{~cm}^{-3}$ and $47 \mathrm{~cm}^{-3}$, respectively.

During ACE-2, a number of discoveries relating to the $\mathrm{CCN}$ instrument were made. Experiences obtained from the field missions serve as the basis for improvements to the current instrument. For example, during ACE-2 water was introduced to the top of the column using a peristaltic pump that forced water to circulate through the column at a constant low flow rate $(<0.5$ $\left.\mathrm{mL} \mathrm{min}^{-1}\right)$. Excess water at the bottom of the column was returned to the water reservoir located below the level of the column. However, it was found that small changes in the water flow rate changed the output diameter substantially, apparently because the flowing water changes the temperature profile along the wet walls and therefore changes the supersaturation profile within the instrument. The revised scheme is to wet the column initially, allow the column to come to steady state, and make measurements with no forced water flow, as described above.

\section{Studies of fixed supersaturation operation}

Because of the limitations of the streamwise gradient technique using this device, we have examined the performance of the instrument as a single supersaturation device. For this mode, the temperature differences between successive segments were held constant. This configuration imposes a supersaturation profile that reaches a constant maximum centerline value. In this mode of operation, the instrument operates as a traditional CCN counter in that particles below a fixed critical supersaturation activate and subsequently grow to sufficiently large enough sizes that they are distinct from particles that remain unactivated. Instrument perfor-

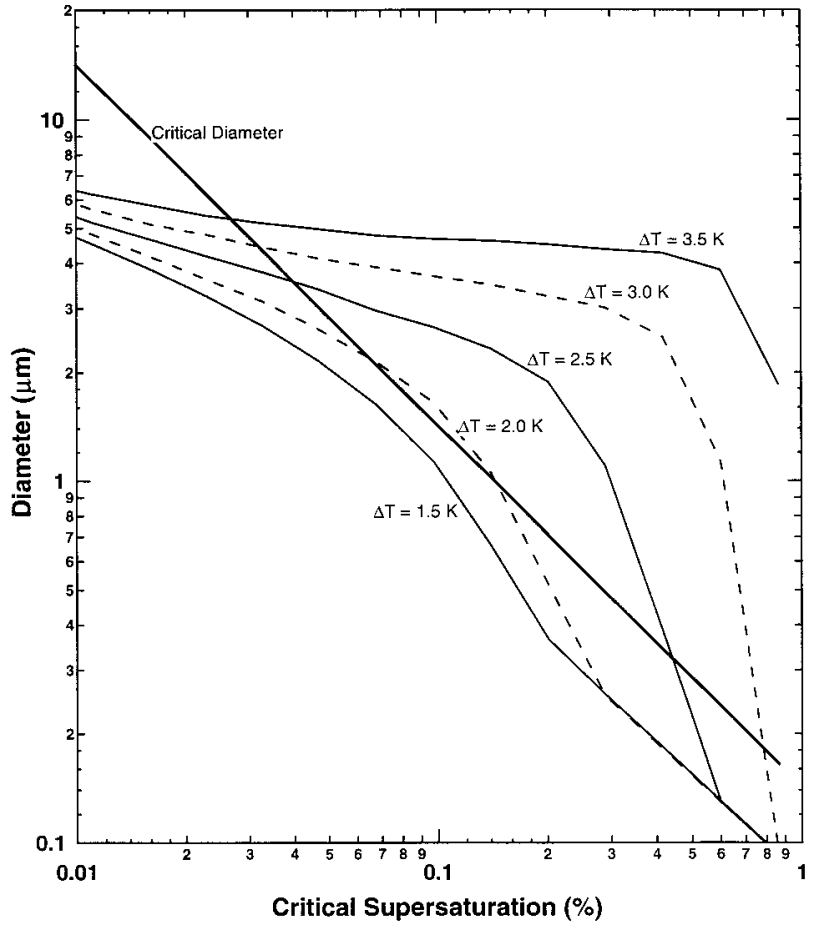

FIG. 8. Simulations of the effect of temperature difference on the final droplet size as a function of critical supersaturation for the fixed supersaturation CCN counter. For all runs, $p=1000 \mathrm{mb}$.

mance in this mode has been studied both experimentally and theoretically.

\section{a. Simulations of single supersaturation instrument}

The instrument has been modeled for various temperature differences and pressures. It is expected that by varying the temperature difference, the instrument can be operated at different fixed supersaturations, with the supersaturation increasing for increasing temperature difference. Pressure variations are examined in order to understand how the instrument characteristics change as aircraft altitude changes.

Figure 8 shows the effect of changing the temperature difference $\Delta T$. For all curves, the temperature of the cold segments is held at $290 \mathrm{~K}$, while the hot temperature segments are adjusted to reach the specified $\Delta T$. When the temperature difference exceeds $2.5 \mathrm{~K}$, the predicted outlet droplet diameter decreases sharply for particles with $S_{c}$ larger than some threshold value. As a result, measurements of the concentration of particles whose size exceeds some threshold size (defined by the lower detection limit of the OPC, estimated to be $1 \mu \mathrm{m}$ for the present instrument) provides a good estimate of the concentration of $\mathrm{CCN}$ below the corresponding critical supersaturation. As the temperature difference is increased, the threshold $S_{c}$ increases as well, and the distinction between activated and unactivated particles sharpens. 


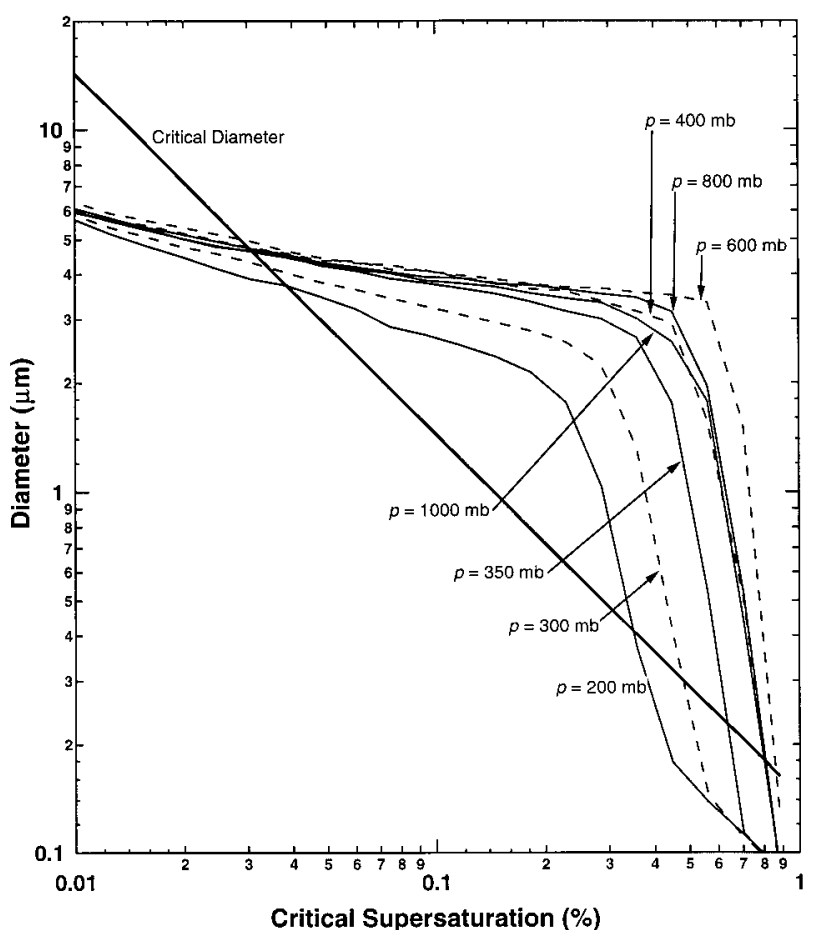

FIG. 9. Simulations of the effect of pressure on the the final droplet size as a function of critical supersaturation for the fixed supersaturation $\mathrm{CCN}$ instrument. For all runs, $\Delta T=3 \mathrm{~K}$.

Figure 9 shows the predicted outlet droplet size distribution as a function of instrument pressure. Dropping the pressure from $1000 \mathrm{mb}$ initially causes an increase in the growth of droplets because the diffusivities of water and heat increase with decreasing pressure, causing the steady-state supersaturation in the instrument to increase. As the pressure decreases further, however, heat and water vapor diffuse so rapidly that each segment more closely approaches equilibrium, lowering the effective supersaturation. As a result, the driving force for condensational growth and the outlet droplet sizes decrease.

The influence of the inlet $\mathrm{RH}$ and total particle concentration was also investigated. The RH of the sheath flow was varied between nearly $0 \%$ and $95 \% \mathrm{RH}$. The size distribution of the droplets at the outlet was measured for a series of monodisperse aerosol sample flows [generated by a differential mobility analyzer (DMA)]. The size distributions were practically identical independent of the sheath flow $\mathrm{RH}$, implying that inlet $\mathrm{RH}$ has little effect on the final droplet diameters. Model predictions are consistent with this result. A humidifier is still desirable, however, to reduce loss of water from the column to the gas phase. The effect of total particle concentration was also investigated by the same method, except that the total concentration of the (monodisperse) aerosol sample flow was varied (and the sheath flow RH was held constant) for a series of inlet particle diameters. No discernable effect was found for particle

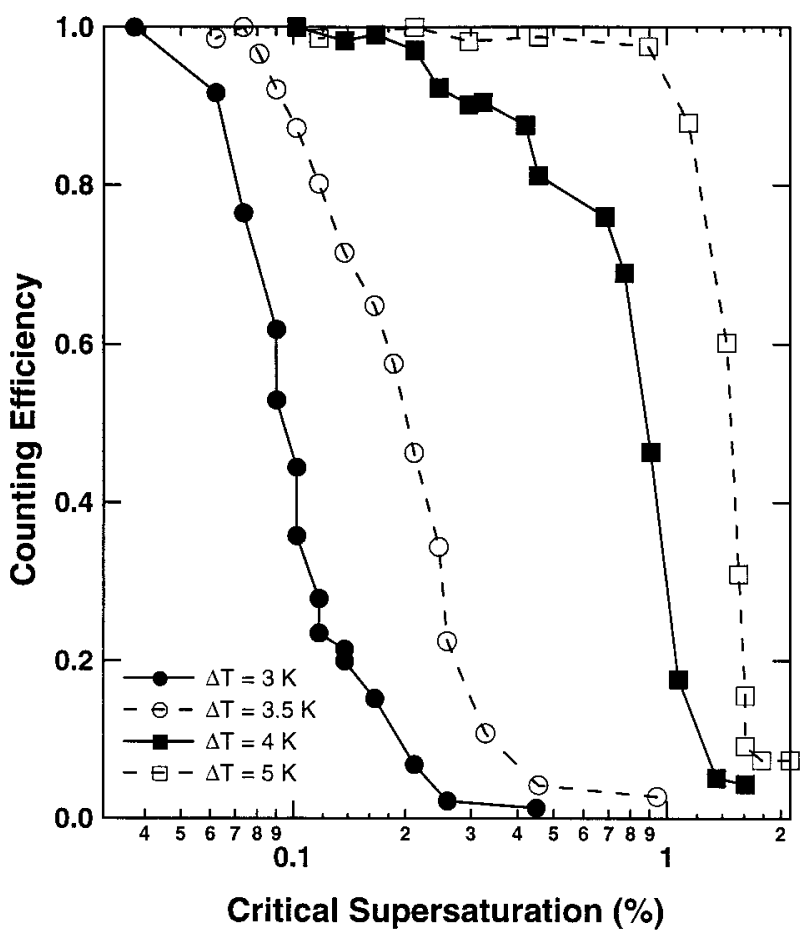

FIG. 10. Results of experiments studying the effect of temperature on the counting efficiency as a function of critical supersaturation for the fixed supersaturation CCN counter. For all runs, $p=1000 \mathrm{mb}$.

concentrations between 10 and $10^{4} \mathrm{~cm}^{-3}$, which is again consistent with model predictions.

\section{b. Experimental studies of the single supersaturation instrument}

The present instrument operated in fixed supersaturation mode was calibrated using monodisperse ammonium sulfate aerosol at a range of particle sizes, temperatures, pressures, and concentrations. A polydisperse aerosol generated by a nebulizer was dried and then classified using a cylindrical DMA to produce a dry, monodisperse aerosol. The monodisperse sample flow was then split between a TSI 3010 condensation nuclei counter (CNC; to measure the concentration of particles in the stream), and the $\mathrm{CCN}$ instrument. An ideal instrument would measure the $\mathrm{CCN}$ concentration to be identical to that measured by the $\mathrm{CNC}$ for particles with critical supersaturation lower than the operating supersaturation of the $\mathrm{CCN}$ instrument, and measure zero $\mathrm{CCN}$ when the particles have a critical supersaturation higher than the operating supersaturation.

Figure 10 shows the variation of the counting efficiency as a function of $S_{c}$ for different values of $\Delta T$. In all cases, the cold temperature segments were maintained at $290 \mathrm{~K}$, while the temperature of the hot segments was changed to give the reported $\Delta T$. The efficiency curves exhibit behavior that qualitatively matches the simulations (Figure 8). As $\Delta T$ increases, the $S_{c}$ 


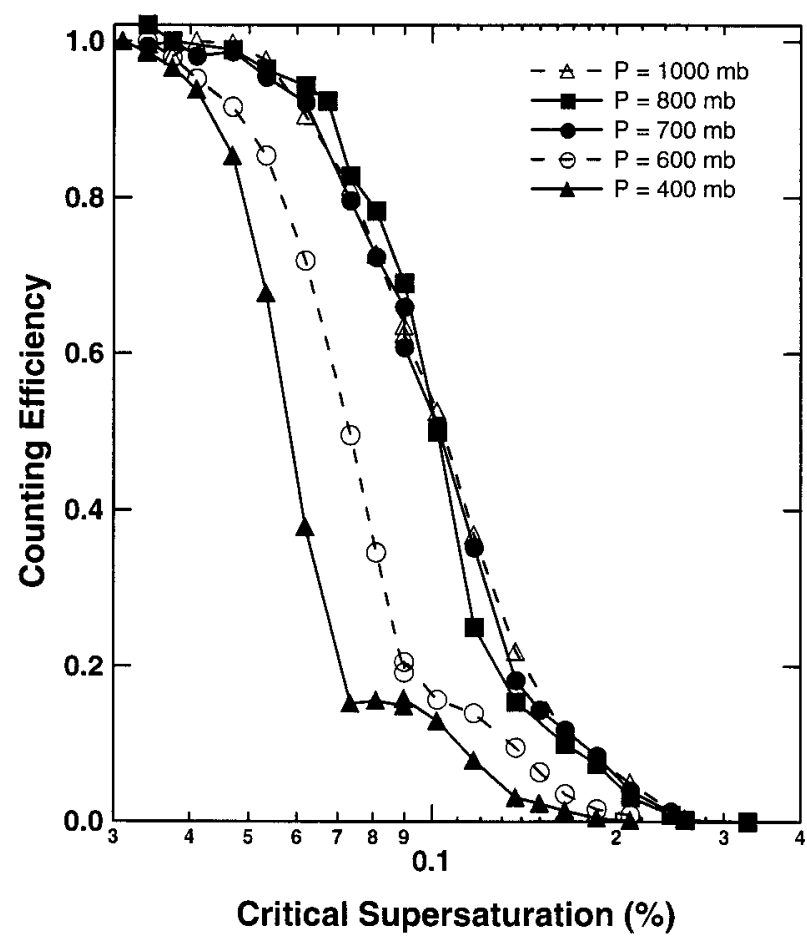

FIG. 11. Results of experiments studying the effect of pressure on the counting efficiency as a function of critical supersaturation for the fixed supersaturation $\mathrm{CCN}$ counter. For all runs, $\Delta T=3 \mathrm{~K}$.

at $50 \%$ efficiency, $S_{50}$, increases, since a larger $\Delta T$ results in a higher supersaturation. It also appears that the curves are more sharply defined at higher $\Delta T$, as is also predicted. For comparison, the curves are normalized to a maximum counting efficiency of unity. Unnormalized, the counting efficiency reaches $95 \% \pm 5 \%$. These curves demonstrate that, because the efficiency curves change sharply from zero to nearly unity, the $\mathrm{CCN}$ instrument can be effectively used as a single supersaturation CCN counter. Simulations of $S_{50}$ do not agree exactly with those measured using the current instrument. The likely explanation for this discrepancy is, again, the temperature difference between the temperature measurement point and the surface of the water film. The actual imposed $\Delta T$ is therefore smaller than that obtained by taking the difference of the temperature set points, accounting for the disagreement between predictions and experimental data.

Because the instrument is designed for airborne measurements, it is important to investigate its behavior as a function of pressure. Figure 11 shows the counting efficiency curves measured during experiments performed at five different pressures. For all five cases, $\Delta T$ was fixed at $3 \mathrm{~K}$. The model predicts that, for pressures greater than $350 \mathrm{mb}$, the variations in $S_{50}$ are small (Fig. 9). The experimental data exhibit somewhat larger changes at low pressures. For pressures $700 \mathrm{mb}$ and above, $S_{50}$ is essentially constant whereas the model predicts a slight increase. At 600 and $400 \mathrm{mb}$, predic- tions suggest that $S_{50}$ should not differ significantly from the $1000-\mathrm{mb}$ value, but the data show decreases of approximately $20 \%$ and $50 \%$, respectively. Again, we speculate that this difference may result from deviations of the temperature profile from that assumed using the model. A complete heat and mass transfer model of the $\mathrm{CCN}$ instrument would be needed to resolve these differences completely, but this is outside the scope of the current study.

One interesting feature of the counting efficiency curves is discontinuity of slope at low efficiency $(\sim 0.15)$, particularly at lower pressures. Ideally, all particles classified by the DMA possess a single charge, permitting unambiguous calculation of their aerodynamic diameter from electrical mobility. In reality, a fraction of the particles carry multiple charges, so larger particles can exhibit the same electrical mobility as the singly charged particles. The ratio of multiple to single charged particles increases as particle size increases, causing the multiple charging problem to be more noticeable at lower $S_{50}$. If the data were corrected for multiple charging, the efficiency curves would reach zero efficiency much more quickly.

Instrument performance can also be altered due to depletion of water vapor. If condensational growth at high $\mathrm{CCN}$ concentration causes a significant decrease in the water vapor concentration, then the supersaturation would decrease, leading to a decrease in $S_{50}$ with concentration. The instrument response was studied for CCN concentrations between 20 and $1300 \mathrm{~cm}^{-3}$, a range that covers the $\mathrm{CCN}$ concentrations expected for most conditions. It was found that the counting efficiency changed very little as a function of $\mathrm{CCN}$ concentration (efficiency standard deviation/efficiency average was 0.07 ), suggesting that water vapor depletion is not a problem for atmospherically relevant $\mathrm{CCN}$ concentrations.

A different experiment was conducted to demonstrate that the current instrument can operate as a single supersaturation CCN counter under conditions in which the sample is a polydisperse aerosol instead of a monodisperse aerosol as used for the above experiments. For this experiment, the DMA system is set up in parallel, rather than in series, to the CCN instrument. Dry polydisperse ammonium sulfate aerosol generated by the nebulizer is used simultaneously as the input for both the CCN instrument and the DMA system. The DMA characterizes the size distribution of the aerosol. Using the efficiency curves calculated for the $\mathrm{CCN}$ instrument and the measured aerosol size distribution, the $\mathrm{CCN}$ measurement can be predicted (using Kohler theory to convert the size distribution into an expected $\mathrm{CCN}$ concentration at a given supersaturation) and compared with the actual measurement made by the fixed supersaturation $\mathrm{CCN}$ instrument. Table 1 shows the results of 10 such experiments performed for a wide range of $\mathrm{CCN}$ concentrations $\left(60-2880 \mathrm{~cm}^{-3}\right)$. The average ratio of measured to predicted $\mathrm{CCN}$ concentration is $0.96 \pm 0.20$ $(1 \sigma)$. For 6 of the 10 experiments, prediction and mea- 
TABLE 1. Results of experiments simultaneously measuring the aerosol size distribution and the $\mathrm{CCN}$ concentration at a single supersaturation. From the measured size distribution, the CCN concentration was predicted and compared to that measured.

\begin{tabular}{lcc}
\hline \hline $\begin{array}{c}\text { Measured } \\
\text { concentration } \\
\left(\mathrm{cm}^{-3}\right)\end{array}$ & $\begin{array}{c}\text { Predicted } \\
\text { concentration } \\
\left(\mathrm{cm}^{-3}\right)\end{array}$ & $\begin{array}{c}\text { Measured/ } \\
\text { predicted }\end{array}$ \\
\hline 60.6 & 60.3 & 1.00 \\
91.7 & 86.9 & 1.05 \\
109 & 110 & 0.989 \\
184 & 188 & 0.977 \\
185 & 238 & 1.30 \\
203 & 156 & 0.776 \\
253 & 364 & 0.695 \\
930 & 813 & 1.14 \\
1380 & 1470 & 0.942 \\
2880 & 4230 & 0.682 \\
Average & & 0.957 \\
$\sigma$ & & 0.196 \\
\hline
\end{tabular}

surement agree to within $6 \%$; all the experiments agree with each other to within about $30 \%$. Some of this variability might be explained by changes in the nebulizer output. The output of the nebulizer is not always constant over the 20 min during which the DMA is characterizing the aerosol size distribution, and any drifting in the source would cause the average size distribution (which is what the $\mathrm{CCN}$ instrument measures) to be different from that calculated using the DMA. This observation is supported by the fact that the cases exhibiting less agreement show no trend with respect to $\mathrm{CCN}$ concentration (which could be explained by water vapor depletion at the very high CCN concentrations), and show no bias for one instrument to systematically measure a higher value than the other.

\section{Summary}

Modeling studies and calibration experiments show that a new streamwise gradient $\mathrm{CCN}$ spectrometer is limited in its capability to resolve supersaturation distributions. Over a climatically important range of critical supersaturations from $0.03 \%$ to $1 \%$, the final particle size varies only by $30 \%$. This limited range is difficult to resolve with the optical particle counter that is used to measure particle size, even though it has been optimized for the refractive index of water droplets.

This CCN instrument does have the ability to accurately measure $\mathrm{CCN}$ concentration at a single fixed supersaturation, and has been shown to be well-suited for unattended airborne measurements, even if it cannot measure the entire supersaturation spectrum simultaneously. The instrument has been shown to give sharp $S_{c}$ cutoff over critical supersaturations ranging from below $0.1 \%$ to over $2 \%$. The continuous-flow instrument can make measurements rapidly $(<10 \mathrm{~s})$, and can be operated autonomously. Its size, weight, and low power consumption make it particularly well suited for measurements aboard small aircraft.
Acknowledgments. This work was supported by Office of Naval Research Grant N00014-91-0119 and National Science Foundation Grant ATM-9614105.

\section{REFERENCES}

Alofs, D. J., 1978: Performance of a dual-range cloud nucleus counter. J. Appl. Meteor., 17, 1286-1297.

— used cloud nucleus counter. J. Appl. Meteor., 15, 350-354.

Berglund, R. N., and B. Y. H. Liu, 1973: Generation of monodisperse aerosol standards. Environ. Sci. Technol., 7, 147-153.

Bohren, C. F., and D. R. Huffman, 1983: Absorption and Scattering of Light by Small Particles. Wiley and Sons, $530 \mathrm{pp}$.

Chuang, P. Y., R. J. Charlson, and J. H. Seinfeld, 1997: Kinetic limitations on droplet formation in clouds. Nature, 390, 594-596.

- , and Coauthors, 2000: CCN measurements during ACE-2 and their relationship to cloud microphysical properties. Tellus, 52B, 843-867.

Delene, D. J., T. Deshler, P. Wechsler, and G. A. Vali, 1998: A balloonborne cloud condensation nuclei counter. J. Geophys. Res., 103, 8927-8934.

Fitzgerald, J. W., C. F. Rogers, and J. G. Hudson, 1981: Review of isothermal haze chamber performance. J. Rech. Atmos., 15, 333346.

Fukuta, N., and V. K. Saxena, 1979: A horizontal thermal gradient cloud condensation nucleus spectrometer. J. Appl. Meteor., 18, 1352-1362.

Gosman, A. D., and A. Ideriah, 1976: TEACH-2E. Tech. Rep. FM83-2, University of California, Berkeley, Berkeley, CA.

Hindmarsh, A. C., 1983: ODEPACK: A Systemized Collection of ODE Solvers. North-Holland.

Hoppel, W. A., S. Twomey, and T. A. Wojciechowski, 1979: A segmented thermal diffusion chamber for continuous measurements of CN. J. Aerosol Sci., 10, 369-373.

Hudson, J. G., 1989: An instantaneous CCN spectrometer. J. Atmos. Oceanic Technol., 6, 1055-1065.

— cloud chamber. J. Appl. Meteor., 15, 776-782.

— , and A. D. Clarke, 1992: Aerosol and cloud condensation nuclei measurements in the Kuwait plume. J. Geophys. Res., 97, $14533-14536$.

-, and G. Svensson, 1995: Cloud microphysical relationships in California marine stratus. J. Appl. Meteor., 34, 2655-2666.

—, Y. Xie, and S. S. Yum, 1998: Vertical distributions of cloud condensation nuclei spectra over the summertime Southern Ocean. J. Geophys. Res., 103, 16 609-16 624.

IPCC, 1996: Climate Change 1995: The Science of Climate Change. Cambridge University Press, 572 pp.

Jiusto, J. E., R. E. Ruskin, and A. Gagin, 1981: CCN comparisons of static diffusion chambers. J. Rech. Atmos., 15, 291-302.

Kandlikar, M., and G. Ramachandran, 1999: Inverse methods for analysing aerosol spectrometer measurements: A critical review. J. Aerosol Sci., 30, 413-437.

Laktionov, A. G., 1972: A constant-temperature method of determining the concentrations of cloud condensation nuclei. Atmos. Oceanic Phys., 8, 672-677.

Lala, G. G., and J. E. Jiusto, 1977: An automatic light scattering CCN counter. J. Appl. Meteor., 16, 413-418.

Leaitch, R., and W. J. Megaw, 1982: The diffusion tube: A cloud condensation nucleus counter for use below $0.3 \%$ supersaturation. J. Aerosol Sci., 13, 297-319.

Patankar, S. V., 1980: Numerical Heat Transfer and Fluid Flow. McGraw-Hill, 197 pp.

Raes and Coauthors, 2000: The second Aerosol Characterization Experiment (ACE-2): General overview and main results. Tellus, 52B, $111-125$. 
Seinfeld, J. H., and S. N. Pandis, 1998: Atmospheric Chemistry and Physics: From Air Pollution to Climate Change. Wiley and Sons, $1326 \mathrm{pp}$.

Shulman, M. L., M. C. Jacobson, R. J. Charlson, R. E. Synovec, and T. E. Young, 1996: Dissolution behavior and surface tension effects of organic compounds in nucleating cloud droplets. Geophys. Res. Lett., 23, 277-280.
Sinnarwalla, A. M., and D. J. Alofs, 1973: A cloud nucleus counter with long available growth time. J. Appl. Meteor., 12, 831835.

Twomey, S., 1963: Measurements of natural cloud nuclei. J. Rech. Atmos., 1, 101-105.

- 1977: The influence of pollution on the shortwave albedo of clouds. J. Atmos. Sci., 34, 149-152. 